\title{
Itinerario terapéutico de los usuarios de una urgencia hospitalar
}

\author{
Itinerário terapêutico dos usuários de uma emergência hospitalar
}

\author{
Liane $M$ aria Lago ${ }^{1}$ \\ Josiane de J esus M artins ${ }^{2}$ \\ Dulcinéia Ghizoni Schneider ${ }^{3}$ \\ Daniela Couto Carvalho Barra ${ }^{4}$ \\ Eliane Regina Pereira do N ascimento ${ }^{1}$ \\ Gelson Luiz de Albuquerque ${ }^{1}$ \\ Alacoque Lorenzini Erdmann ${ }^{1}$
}

${ }^{1}$ Departamento de Enfermagem, Centro de Ciências daSaúde, UniversidadeFederal de SantaCatarina. Campus Universitário Trindade. 88040-970 Florianópolis SC. pongopam@terra.com.br ${ }^{2}$ Hospital Universitário, UniversidadeFederal de

SantaCatarina.

${ }^{3}$ Departamento deCiências Biológicas eda Saúdeede Ciências Sociais A plicadas, Universidadedo Sul deSanta Catarina.

${ }^{4}$ Programa de Pós-

Graduação em Enfermagem, UniversidadeFederal de Santa Catarina.
Abstract This is an exploratory study conducted in a general hospital in the greater Florianopolis in Santa Catarina State, Brazil using a qualitative/quantitative approach. The main objective is to describe the therapeutic route of patients seeking health assistance in an emergency hospital and their justifications for this choice, discussing it primarily from a socio-political and cultural point of view, without the intention to adhere to the theoretical philosophical aspects of these theories. The sample was composed of 35 subjects. The data was collected through semistructured interviews, participant observation and medical charts. The data is presented in tables and using testimonials expressed by the subjects which were categorized by theme and discussed based on references that examine the issue. It was found that $82.6 \%$ of the subjects did not seek assistance at a Basic $\mathrm{H}$ ealth U nit and that thetriageat theemergency hospital was performed by untrained staff. Assistance in emergency wards should be handled systematically and be focused on the user, reassessing and integrating the assistance practices and the work process.

Key words Health systems, Emergency service, H ospital, Triage, U ser embracement
Resumo Se trata deun estudio exploratorio que utiliza el abordaje cualitativo y cuantitativo, realizado en una urgencia de un hospital general en la cercanía de Florianópolis, Santa Catarina, Brasil. El objetivo fue describir el itinerario terapéutico de los usuarios que buscan asistencia en salud en una urgencia hospitalar y las justificativas de esta opción, discutiéndolas de manera preliminar en sus aspectos soci o- políticos y culturales, sin tener la pretensión de atenerse en sustentaciones teórico filosóficas de este aspecto. La muestra fue constituida de 35 usuarios. Para la colecta de los datos, se utilizó la entrevista semi-estructurada, la observación participante y los datos de la ficha de atendimento de la unidad de urgencia. Losdatos fueron presentados en tablas y en forma de hablas expresas por los participantes y agrupados por semejanza de contenido y discutidos por medio de referencias que analizan la temática. Se constató que el $82,6 \%$ de los usuarios no buscaron asistencia en las Unidades Básicas de Salud. El acogimiento y triaje eran realizados por profesionales no capacitados que actúan en la recepción de la urgencia. La asistencia en los servicios de urgencia deberá ser de carácter sistémico y tener como foco el usuario, redefiniendo y integrando las prácticas asistenciales y el proceso de trabajo. Palabras clave Sistema de salud, Servicio hospitalar de urgencia, Triaje, Acogimiento 


\section{Introducción}

LaConstitución Federal Brasileña (1998) establece quela salud es derecho detodosy deber del estado. Para atender a los preceptos constitucionales, fue establecido como modelo de política de salud el Sistema Ú nico de Salud (SUS), representando un marco en la lucha por los derechos de ciudadanía. El desafío permanente del SUS es posibilitar el acceso de todos los ciudadanos de forma universal y ecuánime garantiendo la totalidad, universalidad, humanización y calidad en la atención.

El concepto de salud asegurado en la legislación brasileña se constituye como un derecho de ciudadanía a ser garantizado por el Estado y la universalidad de la atención implica, necesariamente, en la formulación de un modelo social, ético y ecuánimenorteado por la inclusión social y solidariedad humana. En ese sentido, el proble ma vivenciado con la concretización del acceso universal a los servicios de salud requiere una lucha constantepor el fortalecimiento dela salud como un bien público, y dela edificación de una utopía social igualitaria, teniendo la salud como derecho individual y colectivo que debeser fortalecido con el redimensionamiento de una nueva práctica construida desde una gestión democrática y partici pativa ${ }^{1}$.

EI SUS presenta avances importantes, como ampliación del acceso a las acciones de salud, especialmentecon relación a la atención básica. Pero, problemas aún son enfrentados diariamente, principalmente con relación a la oferta desordenada de los servicios quegeneran grandes filas de espera y una demanda reprimida de la atención ambulatorial y hospitalar.

Los Establecimientos Asistenciales de Salud (EAS) son organizaciones prestadoras de servicios en salud, las cuales constantemente utilizan las tecnologías duras, leve-duras y leves ${ }^{1}$. En general, el uso de las tecnologías se limita a las duras y leve-duras, restando las tecnologías leves (relacionales) en un segundo plan. Así, se puede tener el riesgo decentrar el atendimiento en salud apenas en los aspectos biológicos no considerando los aspectos del contexto sociocultural y las necesidades inmediatas del usuario quebusca el cuidado en salud en ese escenario.

En los hospitales, el Servicio de Urgencia (SU) es una unidad importante en virtud de las frecuentes situaciones críticas o de agravantes en salud presente en los usuarios que buscan por el atendimiento ahí ofrecido. Entretanto, en la mayor parte delos casos, el usuario busca esteservicio con problemas desalud que deberían ser aten- didos en la red básica, perdiendo así, los servicios deurgencia, su principal función: atender a usuarios en risco inminente de muerte.

Urgencia implica en una situación crítica que puede ser definida de modo abrangente, como aquella en queel individuo entra en desequilibrio homeostático por enfrentar obstáculos que se anteponen a sus objetivos de vida. Además de eso, una situación de urgencia, también puede ser descrita como alteraciones anormales en el organismo humano, queresultan en drástico trastorno de la salud o en súbita amenaza a la vida, exigiendo medidas terapéuticas inmediatas². Los servicios deurgencia deben estar preparados para prestar asistencia interdisciplinaria en situaciones de este tipo, y no para que se dediquen a consultas de carácter ambulatorial.

Los hospitales que atienden a usuarios en situación de urgencia, en función del ritmo acelerado y constante de trabajo, deben fijarse para la integración de los varios especialistas, actuando con equipes de trabajo coherentes. Así, se torna fundamental la integración entre diferentes categorías profesionales y ramos del conocimiento en torno de un objetivo común, o sea, la asistencia cualificada y libre de riesgos a los usuarios del sistema de salud ${ }^{3}$.

Los servicios de urgencia que prestan asistencia durante las 24 horas del día, y terminan por funcionar como porta de entrada del SUS, acogiendo usuarios en situaciones de urgencia, usuarios con cuadros percibidos como urgencias, usuarios que no recibieron atendimiento en la Unidad Local de Salud y las urgencias sociales. Tales demandas se juntan en las unidades de urgencia, súper Ilenándolas y comprometen a la calidad de la asistencia prestada a la población.

Esta realidad asistencial es, todavía, agravada por problemas sociales organizacionales de éstos servicios como, por ejemplo, la falta detriaje de riesgo, lo que determina el atendimiento por orden de llegada sin evaluación previa del caso, acarreando, muchas veces, graves pérdidas a la salud de los usuarios. Habitualmente, usuarios en estado de urgencias "sangrantes" y con respiración "ruidosas" son priorizadas, pero, infelizmente, es común constatar queusuarios con cuadrosclínicos más graves permanecen horas aguardando por el atendimiento de urgencia, aunque ya esté dentro de este servicio.

El gran número de atendimiento ambulatorial, en las unidades de urgencia, trae como consecuencia un aumento en el tiempo de espera, retrasos en la asistencia urgente y favorece a la elevación de los niveles de estrés de los trabaja- 
dores de estos servicios, exacerbando la vulgarización del sufrimiento ajeno, además de proporcionar un el evado número deusuarios en el sistema de salud de urgencia.

Cuando las personas quedan enfermas, desean un alivio inmediato de sus sufrimientos $y$, para ello, acaban recorriendo a las diversas alternativas curativas existentes, escogiendo lo que mejor les atiende, basado en su cultura, creencias y valores. Por eso, las mismas pueden adherir o no a los tratamientos de salud del subsistema profesional, popular o familiar ${ }^{4}$.

Así, " [...] el itinerario terapéutico no pasa de una colección sintética de proyecciones individuales", o aún, "[...] solamente en el curso de la acción - y, por lo tanto, el acto de elegir - es, queel individuo empieza a interpretar su situación, sus acciones y el estado de cosas resultantes" 5 . La experiencia de la enfermedad, puede ser comprendida como los medios que determinado individuo o grupo social busca soluciones para sus problemas y reproducen los conocimientos existentes en su universo sociocultural 6 . La búsque da por servicios que atenúen el sufrimiento del individuo y que restablezca su proceso de saludenfermedad puede ser comprendida como itinerario terapéutico, y ese, debe considerar también la situación social y cultural de cada ser.

Comprendemos por itinerario terapéutico el camino recogido por el usuario para encontrar diferentes modos de resolver su problema de salud, así como la forma como este elige, evalúa o adhiere a determinado cuidado en salud ${ }^{7}$. Se acreceque esta decisión es influida por el contexto sociocultural en que esteusuario está inmerso, como también por la satisfacción de sus necesidades inmediatas siendo perneados por las posibilidades puestas de acceso al sistema de salud. Las alteraciones vivenciadas por el usuario en su proceso salud-enfermedad también van a influir en la de cisión de éste por determinado servicio de salud.

En el itinerario terapéutico, las personas interpretan ciertos tipos de prácticas como más adecuadas para tratar con ciertos tipos de enfermedades, de tal forma que se considera que los padrones deselección, entre las alternativas terapéuticas, sean definidos por ciertas situaciones deenfermedad, bien como, sus acciones son construidas en un mundo socio - cultural, por medio de un proceso histórico y, por lo tanto, diferente para cada tipo de cultura y sociedad ${ }^{8}$.

La literatura socio-antropológica relata que el itinerario terapéutico [...] tienen como principal objetivo interpretar a los procesos por los cuales los individuos o grupos sociales eligen, evalúan y apli- can 0 no a determinadas formas de tratamiento. Eso se fundamenta en la evidencia de que individuos encuentran diferentes maneras de resolver sus problemas de salud. A mbos afirman que, en estudios referentes a la interpretación del itinerario terapéutico, se torna importante levar en consideración que le elección del tratamiento es influenciada por el contexto sociocultural en que ocurre ${ }^{8}$.

El itinerario terapéutico puede entonces ser definido como la secuencia de actividades quelos individuos buscan para solucionar su problema de salud y se constituyen importante indicador de los recursos sociales, culturales y materiales disponibles ${ }^{9}$. La búsqueda por el tratamiento y su práctica y/o adherencia se relacionan a la historia natural de la enfermedad, bien como al aspecto sociocultural, acordándose que este conjunto es dinámico y mutable. Se destaca todavía, que la percepción del individuo acerca dela condición de salud es factor determinante para la búsqueda de la terapia más apropiada.

El interés de las pesquisidoras por los usuariosquebuscan el atendimiento en el SU emergió desde la observación cotidiana, como docentes y enfermeras asistenciales, y de trabajos ya realizados en esa área. Basadas en experiencias profesionales, las pesquisidoras percibieron la alta demanda de atendimientos que podrían ser solucionados en la red básica de salud. De otro modo despierta la curiosidad sobre el itinerario recogido por los mismos delante dela problemática contextualizada en relación a lafinalidad de eseServicio.

Delante del expuesto, esta investigación buscó contestar la siguiente cuestión: ¿Cuál el itinerario terapéutico recogido por el usuario de los servicios de salud que buscaron atendimiento en una urgencia hospitalar y cual la justificativa de esa decisión del usuario? Para nortear esta investigación, trazamos los siguientes objetivos: describir el itinerario terapéutico de los usuarios que buscaron asistencia en una unidad de urgencia hospitalar y las justificativas apuntadas de esa decisión, discutiéndolas de modo preliminar en sus aspectos socio-políticos y culturales, sin, tener la pretensión deatenerseen sustentación teórico filosófica de estos aspectos.

\section{M etodología}

Se trata de una pesquisa exploratoria de abordajecualitativa-cuantitativa.

El local deinvestigación fueun SU deun hospital de grande porte localizado un municipio cercano a Florianópolis, Santa Catarina, Brasil. 
Los actores sociales escogidos para ser los sujetos de la pesquisa fueron los usuarios de la Unidad de U rgencia de este hospital, particularmente, los usuarios que fueron atendidos en los consultorios de clínica médica y cardiología en los meses de noviembrey diciembre de 2006, totalizando 35 actores sociales, número este considerado suficiente al constatarse la saturación de los datos.

La recolección de los datos fue realizada después de la aprobación del proyecto cop la Comisión de Ética en Pesquisa de la Universidad del Sur de Santa Catarina - CEP/U NISU L, desde el parecer no 06.213.4.04.III y del Comité de Ética del Hospital, recibiendo la aprobación y autorización para su ejecución. Todos los participantes firmaron el termo de consentimiento libre y esclarecido (TCLE) después de ser informados sobre el objetivo de la pesquisa.

Los datos fueron recogidos por medio de entrevista semi estructurada, ficha deatendimiento del usuario en el SU y observación. Se optó por realizar las entrevistas en le ante sala de la triajemédica, logo después la entrada del usuario en la unidad de urgencia. El usuario es llamado a esta sala por un funcionario responsable por la seguridad del servicio de urgencia, de acuerdo a la orden de llegada y después de rellenar la ficha de atendimiento en la recepción del sector. Para eso, fue un guión conteniendo datos relativos a: motivo de la búsqueda del servicio de urgencia hospitalar; Lo qué entiende por Servicio de Urgencia; Se fue atendido en otro local anteriormente(red básica); Q ué situaciones usted considera como atendimiento de urgencia.

La ficha de atendimiento consiste en documento del SU quecontiene informaciones sociodemográficas del usuario, atendimientos anteriores (internaciones 0 atendimiento de urgencia), resumen sucinto de la queja principal del usuario (motivo desu venida al servicio). En este documento es registrado el atendimiento medi$\mathrm{co}$, examines y medicamentos prescritos, siendo rescatada por el sector de cuentas del hospital, para posterior inclusión de los costos hospitalares. Entretanto, se detectaron registros incompletos y de difícil comprensión en las fichas de atendimiento. Los datos por medio de ese instrumento, posibilitó conocer un poco del itinerario terapéutico recogido por el usuario del SU.

Para la observación del camino recogido por el usuario dentro de ese servicio seutilizó un guión conteniendo datos referentes a: tiempo de espe$\mathrm{ra}$; atendimiento realizado en el SU y destino final del usuario.
Los datos fueron presentados en tablas, contiendo frecuencias absolutas y relativas y en forma dehablas expresas por los participantes, agrupados por semejanza de contenido y discutidos por medio de referencias queanalizan la temática y en reflexiones del tema por las investigadoras.

\section{Resultados y discusiones}

Por medio de la caracterización de los actores sociales que constituyeron la muestra, se destaca quelas mujeres $(67,5 \%)$ buscan más atendimiento en el SU que los hombres (34,3\%).

Con relación a la edad de los actores sociales seconstata el predominio de individuos con edad entre 21 y 30 años del sexo femenino y entre 31 y 40 años del sexo masculino. La edad de los actores sociales mayores de 61 años, totalizó solo $25,4 \%$.

Cuanto a la escolaridad, el estudio mostró mayor nivel escolar en el sexo femenino, siendo quelos dos géneros seigualaron en el nivel superior. El nivel de escolaridad entre los sexos fueasí delineado: sexo femenino - analfabeto $5,7 \%$, primaria $34,4 \%$, secundaria $22,6 \%$ y nivel superior $2,9 \%$, totalizando $65,6 \%$; sexo masculino - analfabeto $2,9 \%$, primaria $25,7 \%$, secundaria $2,9 \%$ y nivel superior $2,9 \%$, totalizando $34,4 \%$ delos actores sociales.

Por medio de los datos referentes a la escolaridad se constató que el sexo femenino con nivel escolar más elevado busca el atendimiento de urgencia con mayor frecuencia, totalizando $56,9 \%$. Se presuponeque las mujeres de nivel superior buscan menos el SU debido al nivel deescolaridad implicar en mejores condi ciones sociales, económicas y sanitarias.

Uno de los factores relacionados a la mayor demanda de mujeres en los servicios de salud, se refiere a la cuestion cultural o social, en que ella normalmentees responsablepor acompañar el hijo y los ancianos al medico y frecuentar el prenatal, tornandola más predispuesta a utilizar los servicios de salud. Otro factor es la no existencia de programas de salud volcados específicamente al grupo masculino en el sistema público de salud. Existen cuestiones específicas del hombre, como por ejemplo en cancer depróstatay la violencia que necesitan de esclarecimientos para prevención ${ }^{10}$.

Cuanto al local de residencia de los actores sociales, los datos revelan que $22(62,9 \%)$ viveen la ciudad de São José, 11 (31,3\%) en Palhoça, 1 $(2,9 \%)$ en Biguaçu y 1 (2,9\%) en Florianópolis. Tal hecho justifica que 27 usuarios $(77,2 \%)$ bus- 
caron atendimiento en SU del hospital en estudio visto que viven cerca de él, confirmando que la localiuzación del hospital influyedirectamenteen le elección del servicio de salud.

\section{Categoríasidentificadas}

\section{La búsqueda por atendimiento en salud}

Cuando los actores sociales del estudio fue ron cuestionados se buscaban atendimiento en salud en otro lugar obtuvimos las siguientes respuestas: 6 usuarios $(17,1 \%)$ contestaron quebuscaron el centro de salud, pero no fueron atendidos; 29 usuarios (82,6\%) afirmaron no haber buscado otro servicio de salud y vinieron directamente al SU, una vez que en situaciones anteriores no recibieron cuidados en salud propios para su situación en la red básica.

Los actores sociales que respondieron "haber buscado el servicio básico de salud, pero que no fueron atendidos" expresan las siguientes hablas:

Fui a la sala de salud, pero no había médico;

Allá en la salita no nos dan medicamentosfuertes (significando medicación endovenosa);

Fui al centro de salud y me mandaron a la urgencia;

Necesito de internación;

$N$ unca hay vacante (de antendimiento) en la sala de salud.

Al interpretar la representación de los usuarios que buscaron el servicio de salud en las unidades básicas y que no fueron atendidos $(17,1 \%)$, es preciso considerar la dimensión política que orienta el acceso. El Estado es responsable por el sistema público de salud debiendo garantizar, por medio de la distribución planeada de recursos, la localización geográfica de la unidad (el hospital está más cerca), la disponibilidad de los servicios (usuario busca, pero cuando llega al servicio básico de salud no encuentra atendimiento) y la articulación entre los servicios que componen la red, de acurdo con las demandas y necesidades de la población. Por lo tanto, la realidad observada se integró al cenário brasileño una vez que ella no incorporó las matrices básicas que sirven de suporte para un modelo de salud publico y universal conforme establece el SU $S^{11}$.

Queda claro en la hablas de los usuarios que buscar primeramenteun SU es garantizar el atendimiento desu necesidad inmediata de salud, aunque esta no se encuadre en los criterios de atendimiento de una urgencia. Así, el análisis sobre el itinerario terapéutico involucra necesariamente la idea dequelas distintas trayectorias individuales se viabilizan en un campo de posibilidades socio-culturales, que permiten la elaboración y implementación de proyectos de tratamiento específicos 0 hasta mismo contradictorios? ${ }^{7}$. Las contradicciones existentes en el itinerario de búsqueda por el cuidado en salud de este usuario refuerza la necesidadde acciones que amplien las discuciones sobre las reales funciones de las unidades local es de salud y cómo estas están organizadas para atender las necesidades de los usuarios que residen en el area de abrangencia de estas.

\section{Estar con problemas de salud}

En relación a los problemas de salud, 19 $(54,3 \%)$ refieren poseer otras enfermedades y 16 $(45,7 \%)$ afirman no tener. Entre los problemas de salud relatados, $52,6 \%$ son portadores de $\mathrm{Hi}$ pertensión Arterial Sistémica (HAS); 15,8\% HAS y Diabetes M ellitas (DM ); 15,8\% dolor crónicay; $15,8 \%$ otros problemas tales como, infección urinaria de repetición, alergias, cardiopatía, problemas articulares y varices.

Las enfermedades crónicas atingen casi un tercio de la población brasileña. DM , H AS, cáncer, problemas osteoarticulares están entre las enfermedades crónicas que acometen 52,6 millones de brasileños ( $29,9 \%$ de la población), de los cuales 22,1 millones son hombres y 30,5 millones mujeres. En el total, 9,7 millones de individuos tienen tres 0 más enfermedades crónicas ${ }^{12}$.

Un aspecto que merece destaque es el SU ser utilizado para atendimiento de usuarios portadores de enfermedades crónicas, cuyo acompañamiento y cuidado debería ser realizado en ambulatorios. Pero la agudización de una enfermedad crónica lleva el usuario a buscar el SU por saber previamente que en la red básica hay pocos recursos disponibles. Esta situación no es coherente con el cambio del modelo asistencial pretendido por el SUS y también con las necesidades de los portadores de problemas crónicos de salud. Si la red básica estuviese debidamente preparada para atender estos usuarios la demanda de los servicios de urgencia disminuiría considerablemente.

Se destaca queel camino recorrido por el usuario para atender su necesidad de salud, siempre considerará sus experiencias sociales y culturales, así al establecer sus prioridades de cuidado, estas, pueden discrepar de las prioridades establecidas por los servicios de salud profesionales ${ }^{13}$. 
Significado de urgencia

y SU para los usuarios

La totalidad de los actores sociales comprende por urgencia una situación grave en e estado de salud de uno, como accidentes, infarto agudo del miocardio (IAM), accidentevascular cerebral (AVC). La unanimidad en las respuestas mostró el conocimiento de la realidad urgente por el entrevistado, entretanto, delante del atendimiento que seguramente recibieron, es decir, la evaluación clínica, los examenes solicitados y la medicación administrada, asociado a la proximidad del local, ponen la unidad de urgencia en evidencia por su alta capacidad de solución y caracteriza la pequeña búsqueda por la red básica.

La asistencia en situaciones de emergencia y urgencia se caracteriza por la necesidad de un usuario ser atendido en un curto espacio detiempo. La emergencia es definida como la situación en queel atendimiento no puede tardar, es decir, la asistencia debe ser inmediata. En las urgencias el atendimiento debe ser prestado e un periodo de tiempo que, en general, es considerado como no superior a dos horas. Las situaciones no urgentes pueden ser referidas para el pronto atendimiento ambulatorial o para el atendimiento ambulatorial convencional, una vez que no hay las características ya descritas anteriormente.

Siendo así, en las situaciones de atendimiento de emergencia o urgencia el criterio de acceso a los servicios es el de la gravedad. De acuerdo a este criterio, los usuarios en situación de urgencia son atendidos en primer lugar. Muchas veces pacientes en situaciones no urgentestambién provocan este tipo deservicio por se, teóricamenteel más disponible. Se sabe también que muchos usuarios no tienen otros servicios de salud para recurrir en el momento en quela enfermedad los fragiliza. Frente a esta situación difícil, michas veces el profesional desalud atiendeeste usuario, consciente de que esta destorciendo el objetivo del servicio. Esto puede provocar otra situación difícil, que es la no disponibilidad de recursos emergenciales para los pacientes que efectivamentenecesitan de este atendimiento.

Como situaciones de salud consideradas "no urgente", 14 (40\%) de los actores sociales respondieron fiebre, gripe, dolor de poca intensidad; 6 $(17,1 \%)$ destacan la fiebre, la diarrea y el dolor abdominal; 5 (14,3\%) gripe, dolor leve; 3 (8,3\%) presión arterial alterada y DM ; $3(8,3 \%)$ no supieron explicar y, $4(11,4 \%)$ no respondieron. Desde esta cuestión, las respuestas obtenidas revelas que el estado febril, gripe y dolor no serían los factores principales dela búsqueda por el atendimiento emergencial en una unidad hospitalar.

Percepción de los usuarios

sobre el atendimiento en el SU

Los actores sociales fueron cuestionados acerca del atendimiento recibido en el SU. Las respuestas demuestran satisfacción de los usuarios cuanto al atendimiento prestado por el SU conforme explicitado a seguir: $12(37,1 \%)$ consideran el atendimiento excelente; $20(57,1 \%)$ atendimiento bueno; 1 (2,9\%) atendimiento rápido; 1 $(2,9 \%)$ no contestó.

La evaluación en salud tienecomo presupuesto una verificación de eficiencia, eficacia y efectividad de las estructuras, procesos y resultados relacionados al riesgo, acceso y satisfacción de los ciudadanos delante de los servicios públicos de salud en la búsqueda de la solución y calidad.

La evaluación es, en especial, partefundamental en el planeamiento de la gestión del SUS, basándoseen el presupuesto dequeun sistema efectivo deevaluación debereordenar la ejecución de las acciones y servicios, redimensionándolos de forma a contemplar las necesidades de su publico, dando mayor racionalidad al uso de los recursos, visando así, analizar como está el atendimiento al usuario y la estructura del establecimiento. La evaluación debeser realizada también junto al trabajador del SUS, con preguntas sobre su inmersión en la institución, su capacidad, el nivel de atendimiento ofertado y el tipo de respuesta que él recibe del usuario ${ }^{14}$.

Por medio de las fichas de atendimiento de los usuarios en el SU y de la observación fue posible detectar el tratamiento recibido por los mismos y el destino final de la asistencia prestada en ese servicio. Se verificó que 31 (88,6\%) fueron encaminados para la sala de medicación y posteriormente recibieron alta hospitalar y 4 usuarios $(11,4 \%)$ permanecieron en observación y/o fueron encaminado para internación hospitalar (el término "observación" se refiere al período de tiempo que transcurre del término de la consulta inicial hasta el alta hospitalar o hasta la internación en una delas unidades del hospital. Durante el período de observación el usuario ocupa uno de los lechos de la unidad de urgencia).

La utilización de los servicios en salud comprende, por lo tanto, diferentes deshechos, incluyendo consultas médicas curativas, preventivas y internaciones. El estudio del padrón de utilización de servicios de salud por la población es indispensable para el planeamiento e desenvolvi- 
miento de acciones que busquen reducir las desigualdades en el adolecer y en el acceso a los servicios de salud.

El atendimiento en las urgencias, es un termómetro del funcionamiento del SUS y de las posibles fallas de éste. Es en la puerta de los SU que más se evidencia los principales obstáculos y las fallas de la atención primaria en salud, traducidos aquí como la no accesibilidad universal, la no equidad, la miseria social y la agudización de la desasistencia de la población, es decir, el desprecio de la salud pública por las diferentes instancias de gobierno y el descaso con la vida de la población usuaria del SU ${ }^{15}$.

M ediante los datos recaudados, fue hecho el itinerario terapéutico del atendimiento ofrecido al usuario en el SU : 1 o recepción; 20 triaje realizada por profesionales no capacitados; 3 - atendimiento clínico con especialista (cardiólogo, ortopedista, clínica médica); 40 administración de medicamentos y/o solicitación de exámenes; 50 después de la consulta médica el usuario recibe alta hospitalar o es encaminado para la observación; 6o observación o internación hospitalar.

Con relación al itinerario terapéutico recogido por el usuario antes de buscar el SU dos caminos fueron trazados: a) 10 búsqueda por la red básica sin solución y 2o búsqueda por el SU.

Este itinerario es retratado en las hablas que siguen:

Prefiero venir derecho a la urgencia, el atendimiento es má rápido. Puede demorar algunas veces, pero la gente sale con tratamiento y exámenes. (U14)

En la unidad de salud todo es más difícil, nunca tiene nada. $\mathrm{H}$ ay que marcar médico, no tiene medicinas ni como hacer radiografía o electrocardiograma. (U 18)

Aquí en la urgencia, aunque demore, la gente es atendida. (U 31)

A veces es mejor venir derecho a la urgencia que estar esperando en la sala de salud (Unidad Básica de Salud). Hubo ocasiones en que estuve muy mal, pero hasta hoy espero quela sala desalud mellame. No funciona. (U 31)

Hay, sin duda, una justificativa aceptable para la opción de buscar el SU. Todavía, no se puede desconsiderar la costumbre de haber siempre buscado al servicio de urgencia, una vez que en ella encontraron la respuesta inmediata al problema. La concepción de salud y los límites de reconocimientos de sus posibles agraves hacen parte del modelo de pensar y actuar de los grupos sociales. Los aspectos culturales son de cierto modo, determinantes de las actitudes de las personas delante de sus problemas de salud y de cómo la red social está íntimamente imbricada en los encaminamientos o trayectorias recogidas por los usuarios.

La enfermedad involucra cuestiones intelectuales, culturales y prácticas y, a lo largo de este proceso, el usuario opta por determinados caminos, como también abandona otros, una ves que su objetivo mayor es tratar su enfermedad. El itinerario se construyeo es construido deacuerdo con el significado atribuido a enfermedad, y a las diferentes maneras de enfrentarla ${ }^{7}$.

\section{Consideraciones finales}

El objetivo deéstetrabajo fue describir el itinerario terapéutico recorrido por los usuarios que buscan asistencia en salud en un SU hospitalar, asó, por medio de 35 entrevistados, se presentó los motivos princi pales deesta búsqueda y cuales caminos fueron recorridos por los pacientes, antes de su llegada a la unidad de urgencia, y cual su destino después del atendimiento dentro dela institución y sus percepciones del servicio.

La mayor parte de los entrevistados $82,6 \%$ no fue buscar asistencia en las unidades básicas de salud, que en principio tenían la indicación como primera opción, aunque la queja principal fue dores ortopédicas (en las unidades básicas de salud no hay especialista ortopedista). Entretanto, se percibió que en los encaminamientos después de la consulta clínica, estos fueron encaminados directamente a la sala de medicación, y no al servicio de ortopedia, significando así, que el profesional de una unidad básica tendría la misma conducta.

Aunqueseidealiceun modelo deatención ala salud, con base en la promoción de la salud, se sabe que el SUS todavía sigue, de forma más intensa, actuando y organizando acciones de salud con el objetivo de atender aquello que buscan por asistencia médica (ambulatorios, centros de salud) y hospitalar (urgencia y emergencia), no teniendo una implantación satisfactoria en términos de atendimiento en la red básica. Por eso, la asistencia hospitalar ha sido la puerta de entrada para el SUS, favoreciendo un mayor número de atendimientos y internaciones que podrían ser evitadas, caso hubiese una mejor práctica de la atención primaria a la salud.

Este estudio, de cierto modo, muestra la forma deacogimiento en la recepción del SU, en que funcionarios de la recepción y de seguridad del sector definen la prioridad del atendimiento, desde 
la queja principal del usuario. Tal vez aquí, se identifique el aspecto mas grave del atendimiento en eses servicios, cuando el individuo, para además de las condiciones del ambiente (estrés, conforto, etc.), queda expuesto a un criterio espurio de selección de sus problemas, en que los casos más graves pueden no ser priorizados.

El camino para superar el modelo actual de atención en los servicios de urgencia deberá ser de carácter sistémico y tener como foco el usuario, con redefinición eintegración delas vocaciones asistenciales, reorganización de flujos y repactuación del proceso de trabajo. El desafío es hacer funcionar el SUS con todo su potencial de calidad. Al planear la asistencia a las situaciones de urgencia y emergencia es necesario tener el conocimiento del que, con cual gravedad y a quienes se atiende, cuanto tiempo se usa para estos atendimientos y el destino de los pacientes.

Así, se refuerza la importancia de la formulación y implementación de políticas que presenten resultados prácticos para la población con relación a la seguridad, a la cultura, a la educación, al pasatiempo, a la interacción social y a las oportunidades de trabajo, con significanteincremento en los niveles de calidad de vida y desalud de los ciudadanos.

Investigaciones deben ser hechas abordando esta temática, para así posible comprender las vacunas sociales y culturales que pernean los cuidados en salud ofrecidos por los diversos servicios, una vez que podrá favorecer la interacción del usuario con los trabajadores y servicios.

Importante destacar también que somos responsables por estos usuarios que buscan los servicios de salud y muchas veces banalizamos el sufrimiento de este ser. Actuando de este modo, contribuimos para la propagación del sufrimiento ajeno. Somostambién victimas deestesistema de salud, entretanto estamos del otro lado, ofrecemos y realizamos los cuidados en salud. Es pertinente cuestionar: ¿Cuál es nuestra co-responsabilidad con el usuario de los servicios de salud? ¿Respetamos su subjetividad? ¿Estamos también perdiendo la esperanza por el sistema que creemos y defendemos? Así es imprescindible pensar en estrategias colectivas para cambiar este escenario re-significando nuestras acciones.

\section{Colaboradores}

LM Lago trabajó en la concepción, delineamiento, análisis e interpretación de los datos. JJ M artins fue la coordinadora de la investigación, trabajó en la concepción, delineamiento, análisis e interpretación de los datos. DG Schneider colaboró con la investigación, trabajó en el análisise interpretación de los datos. DCC Barra trabajó en la coleta de los datos y análisis. ERP Nascimento trabajó en el delineamiento y metodología. GL Albuquerque trabajó en la concepción y redacción final. AL Erdmann trabajó en la concepción, análisis y redación final. 


\section{Referências}

1. Assis MA, Villa TCS, Nascimento MAA. Acesso aos serviços de saúde: uma possibilidade a ser construída na prática. Rio de Janeiro: Abrasco; 2006.

2. Neves C. Urgências e emergências em saúde: perspectivas de profissionais e usuários. Cad Saude Publica 2006; 22(3):691-693.

3. Alves M, Ramos FRS, Penna CMM. O trabalho interdisciplinar: aproximações possíveis na visão de enfermeiras de uma unidade de emergência. Texto Contexto Enferm 2005; 14(3):323-331.

4. Helmann CG. Cultura, saúde e doença. Porto Alegre: Artmed; 2003.

5. Alves PCB, Souza IAM. Escolha e avaliação de tratamento para problemas de saúde: considerações sobre itinerário terapêutico. In: Rabelo M CM, Alves PCB, Souza IMA, organizadores. Experiência de doença e narrativa. Rio de Janeiro: Fiocruz; 1999.

6. Alves PC. A experiência da enfermidade: considerações teóricas. Cad Saude Publica 1993; 9(3):263271.

7. Reinaldo AMS. Conhecendo o itinerário terapêutico em saúde mental pela história oral de vida do paciente psiquiátrico [dissertação]. Ribeirão Preto (SP): Escola de Enfermagem de Ribeirão Preto, USP; 2003.

8. Mattosinho M MS. Itinerário terapêutico do adolescente com diabetes mellitus tipo 1 e seus familiares [dissertação]. Florianópolis (SC): Programa de PósGraduação em Enfermagem, U FSC; 2004.

9. Kleinman A. Patientes and healers in the context of culture. Berkeley: University of California Press; 1980.

10. Laurenti R, Jorge MHPM, Gotlieb SLD. Perfil epidemiológico da morbi-mortalidade masculina. Cien Saude Colet 2005; 10(1):35-46.

11. Barros E. Política de saúde no Brasil: a universalização tardia como possibilidade de construção do novo. Cien Saude Colet 1996; 1(1):5-17.

12. Instituto Brasileiro de Geografia e Estatística. Doenças crônicas atingem quase um terço da população brasileira. [acessado 2007 fev 20]. Disponível em: http://www.ibge.com.br/home/presidencia/noticias/ noticia_visualiza.php

13. Munõz AMA, Herrera ACV. Dimensiones culturales en el proceso de atención primaria infantil: perspectivas de las madres. Salud pública de M éxico 2005; 47(6):440-446.

14. Brasil. M inistério da Saúde. Secretaria de Assistência à Saúde. Departamento de Normas técnicas. $\mathrm{N}$ ormas para estabelecimentos assistenciais de saúde. Brasília: Ministério da Saúde; 1995.

15. Giglio-Jacquemot A. U rgências e emergências em saúde: perspectivas de profissionais e usuários. Rio de Janeiro: Fiocruz; 2005. 